\title{
Nachruf auf Carl Nedelmann
}

\section{Ulrich Lamparter}

Online publiziert: 2. September 2016

(C) Springer-Verlag Berlin Heidelberg 2016

Am 20. Juni 2016 ist der Mitherausgeber des Forum der Psychoanalyse Carl Nedelmann in Hamburg im Alter von 80 Jahren verstorben. Carl Nedelmann war seit 1995 Mitherausgeber des Forum der Psychoanalyse. Er stellte sich dieser Aufgabe mit Freude, Zuverlässigkeit und Klarsicht. Bis in seine letzten Tage hinein galt seine Sorge den ihm anvertrauten Manuskripten. Er repräsentierte in der Gruppe der Herausgeber die Stimme des klugen Mahners, eines für die Nöte des Autors verständnisvollen Textbearbeiters und nicht zuletzt des Bewahrers der zentralen Inhalte der Psychoanalyse.

Die Psychoanalyse war ihm nicht nur ein erfolgreiches Therapieverfahren oder allgemeines Kulturgut, das sich im Wettstreit mit anderen psychotherapeutischen Verfahren und Gesellschaftstheorien zu behaupten hatte, sondern ein Mittel zum psychischen Überleben. Hier war er ganz Kriegskind, den Verstörungen, Irritationen und Umwälzungen eines 1936 Geborenen ausgesetzt. Aufgewachsen in begüterten Verhältnissen, der Vater Direktor einer chemischen Firma am Mittelrhein, stand er lebenslang unter dem Eindruck der Ausbombung, der Zerstörung des Hauses und der damit verbundenen Traumatisierung. Seine geliebte Freundin kam im Nachbarhaus um. Die Flieger hatten Wohnhäuser angegriffen, anstatt das kriegswichtige wenige Kilometer entfernte Hydrierwerk zu bombardieren. Rettung war für ihn das Erleben von Beziehung in der Provinz, wo ein Teil der Familie Zuflucht gefunden hatte.

Im Laufe seines Jurastudiums fand er Hilfe in der Psychoanalyse. Er begann, Medizin zu studieren, und wurde Psychoanalytiker. Die Freude am Wort und der scharfsinnigen Auseinandersetzung hatte er sich bewahrt. Noch als Student gab er mit dem späteren Verfassungsrichter Gert Schäfer ein Buch über die Verfassungs-

PD Dr. med. Dipl.-Psych. U. Lamparter ( $₫)$

Poliklinik für Psychosomatische Medizin und Psychotherapie, Zentrum für Innere Medizin,

Universitätsklinikum Hamburg-Eppendorf, Martinistr. 52, 20246 Hamburg, Deutschland

E-Mail: lamparter@uke.de 
wirklichkeit der Bundesrepublik Deutschland heraus: Der CDU-Staat (Schäfer und Nedelmann 1969). Im Jahr 1969 in der Reihe der Suhrkamp-Taschenbücher erschienen, kann dieser Band als Wetterleuchten der Studentenbewegung gelten und brachte den Verfasser auf die Beobachtungsliste des Verfassungsschutzes. Spätere Erfahrungen in der psychoanalytischen Ausbildung sensibilisierten ihn lebenslang gegen Anmaßungen und Selbstherrlichkeiten des Analytikers im Umgang mit Fragen des Settings.

Seine offenkundige Begabung führte zu einem Angebot von Wolfgang Loch in Tübingen, bei ihm Oberarzt zu werden. Lochs Denken und sein praktisches Vorbild haben bei Carl Nedelmann anhaltend wahrnehmbare Spuren hinterlassen. Einmal fragte er den Meister, was er denn mache, wenn er zeitgleich mit dem Patienten eintreffe und die lange schmale Treppe des in der Neckargasse gelegenen Instituts hinaufsteige, ... wie seien hier Peinlichkeiten zu vermeiden? Der Meister: „Dann unterhalte ich mich ganz einfach mit dem Patienten." Nedelmann schrieb, wie er sprach: glasklar und eindeutig. Manchmal lagen die Bedeutungen in den Auslassungen und Übergängen, wenn er kurze, ausgefeilte mit reichem Inhalt gefüllte Sätze aneinanderreihte. Mit seinem Lehrer Wolfgang Loch teilte er die Freude an der lebendigen Begegnung nicht zuletzt in der Balint-Gruppe und die Orientierung an der Objektbeziehung. Bei Carl Nedelmann tritt ein wesentliches Element hinzu: seine unablässige Aufforderung, der „Realität“ Aufmerksamkeit zu schenken und so das freudsche Diktum aufzugreifen, dass die Übereinstimmung mit der Realität Wahrheit sei. Andere wichtige Lehrer in der Psychoanalyse waren Helmut Thomä und Paul Parin.

Im Jahr 1983 wurde Carl Nedelmann zum Direktor des damals noch staatlichen Michael-Balint-Instituts in Hamburg berufen. Realität bedeutete im Denken von Carl Nedelmann nicht nur die berufspolitische Auseinandersetzung. Hier hat er als Vorsitzender der Deutschen Gesellschaft für Psychoanalyse, Psychotherapie, Psychosomatik und Tiefenpsychologie (DGPT) von 1983 bis 1985 und der Deutschen Psychoanalytischen Vereinigung (DPV) von 1992 bis 1994 wichtige Weichen gestellt.

Besonders lag ihm der Kontakt mit jüdischen Psychoanalytikern und Überlebenden der Shoah am Herzen, namentlich Hillel Klein, den er zu einem bewegenden Vortrag bei der DPV einlud. Realität hieß für Carl Nedelmann eben nicht nur psychische Realität, sondern auch Geschichte, und ganz besonders die unteilbare deutsche Geschichte und die Shoa. Nach seiner Auffassung hatten sich Psychoanalytiker nicht nur um das Innerste des Menschen zu kümmern, sondern auch um den großen Zusammenhang der Welt und ihre realen Bedrohungen. Mit großer Sorge verfolgte er das Anwachsen der nuklearen Bedrohung mit ihrem ganzen Irrwitz unter dem Deckmantel einer vorgeblichen Rationalität und engagierte sich in den 1980erJahren führend in der Internationalen Vereinigung der Psychoanalytiker gegen den Atomkrieg. Er nahm persönliche Verbindungen in die damalige „Ostzone“ auf, als noch kaum jemand nach Weimar oder Leipzig fuhr. Bis zuletzt unterhielt er eine reichhaltige Vortrags- und Publikationstätigkeit. Auch als gesuchter Lehranalytiker engagierte er sich in der psychotherapeutischen Ausbildung und suchte Integration ohne Abstriche vom Wesentlichen. 
Wo Carl Nedelmann war, war lebendige Auseinandersetzung. Nicht alle konnten ihm folgen, und er folgte nicht allen. Seine dialektischen Wendungen zwischen Progression und Bewahrung waren nicht jedem nachvollziehbar. Wenn er sich entschieden hatte, hatte er sich entschieden. So trug er fortwährend denselben geerbten Mantel seines Vaters von höchster Qualität, und kaum ein Kollege wird ihn nicht im grauen Anzug erlebt haben. Den revolutionären Look der 68er oder das grüne Outfit vermied er. Er erklärte mir, dass man sich konservativ anziehen müsse, sonst werde man nicht ernst genommen. Seiner Rotariergruppe aus dem vornehmen Hamburger Stadtteil Rotherbaum verordnete er eine Reise in die Internationale Jugendbegegnungsstätte nach Auschwitz, der seine treue Zuneigung galt. Er focht nicht mit dem intellektuellen Säbel, sondern warb mit der Stimme der Vernunft; er antizipierte lieber mögliche Entwicklungen, anstatt sich mit Verve in die Linien der Auseinandersetzung zu werfen oder rein taktische Bündnisse zu schließen. Er ließ zu, wenn anders gedacht wurde, und man verlor nicht seine Wertschätzung, wenn man eigene Wege ging. Er hielt viel aus, vielleicht manchmal auch zu viel. Vielleicht vermied er aus einer scheuen und diskreten Grundhaltung manches Mal das Unmittelbare und machte sich nicht gemein, was manche spürten und Grenzen im Kontakt einhalten ließ.

Die letzte Periode seines Schaffens war dem Begriff der Sublimierung gewidmet, den er am Beispiel einer unerfüllten Liebe Goethes zur Herzogin Amalie von Weimar aus dem Schattendasein herausholte. Seine eigene Freude an den Gedichten aus der Sammlung West-östlicher Diwan belegte seine Thesen zur Sublimierung eindrucksvoll. Sie gab seinen Schülern und Freunden hoffnungsvolle Zeichen für ein erfülltes Leben im Alter trotz seiner Gebrechen und Limitierungen. Wie er dem unausweichlichen Tod nüchtern und klar entgegentrat, erfüllte seine Angehörigen und Freunde mit Bewunderung und tief empfundenem Respekt. Der Verlust von Carl Nedelmann wiegt schwer. Für viele ist die Welt nach seinem Tod eine andere geworden. 